\title{
GENETIC COUNSELLING IN X-LINKED OCULAR ALBINISM: Clinical features of the carrier state
}

\author{
S. J. CHARLES, A. T. MOORE, J. W. GRANT, J. R. W. YATES \\ Cambridge
}

\begin{abstract}
SUMMARY
Forty-nine obligate heterozygotes and 64 at risk females were assessed from 17 families affected by $\mathrm{X}$-linked ocular albinism to determine the relative values of clinical examination and skin biopsy in carrier detection. $92 \%$ of obligate heterozygotes had a mud-splattered appearance of the fundus with hyperpigmented streaks and in $\mathbf{7 4 \%}$ this was associated with marked iris translucency. Skin histology showed macromelanosomes in $84 \% .27$ of 64 $(42 \%)$ at risk females had definite features of the carrier state and $19(30 \%)$ were unequivocally normal but a further $18(28 \%)$ had mild RPE abnormalities, often with iris transillumination defects, of uncertain significance. Fundus examination will detect most carrier females but will not allow reliable genetic counselling for all at risk females, even when supplemented by skin biopsy.
\end{abstract}

Albinism is a cause of poor visual acuity and nystagmus and can be broadly divided into two groups: ${ }^{1}$ oculocutaneous albinism, of which there are several types, characterised by hypomelanosis of the hair, skin and eyes, which has an autosomal recessive inheritance; and ocular albinism where the hypomelanosis appears confined to the eyes. Ocular albinism may occur in an autosomal recessive form (AROA) ${ }^{2}$ but more commonly it shows $X$-linked inheritance ${ }^{3}$ and is known as Nettleship-Falls or X-linked ocular albinism (XLOA).

XLOA has a prevalence of approximately $1: 50,000{ }^{4}$ Affected males have reduced visual acuity (6/18 to 6/60), nystagmus, strabismus, refractive errors, iris translucency, fundus hypopigmentation and foveal hypoplasia. Abnormal decussation of the optic chiasm has been demonstrated by visual evoked potentials. ${ }^{12}$ Although effects were initially thought to be confined to the eyes, skin histology in affected males shows abnormal giant melanin granules ${ }^{5,6}$ termed macromelanosomes. These were initially thought to represent an aberrant pathway in melanosome morphogenesis, ${ }^{7}$ but may represent forms of autolysosomes ${ }^{8}$ rather than true macromelanosomes.

Correspondence to: Mr Stephen Charles FCOphth, Department of Ophthalmology, Addenbrooke's Hospital, Cambridge CB2 $2 \mathrm{QQ}$.
Macromelanosomes may also be found in ChediakHigashi disease, ${ }^{9}$ naevus spilus, ${ }^{7}$ neurofibromatosis, ${ }^{10}$ and xeroderma pigmentosum ${ }^{11}$ but not in normal skin.

Most carrier females have normal vision but may be detected on the basis of iris translucency (Fig. 1) and a characteristic mud-splattered fundus appearance ${ }^{13}$ (Fig. 2). Some obligate carriers are described with an entirely normal ophthalmic examination..$^{14}$ In rare cases carriers show reduced visual acuity and nystagmus, ${ }^{15,16}$ being manifesting heterozygotes. The skin of carrier females may also contain macromelanosomes (Fig. 3) and it has been suggested that skin biopsy may be a more sensitive way of detecting carrier females than ophthalmic examination. ${ }^{17}$

There are two particular situations where the diagnosis of XLOA may be problematic. Daughters of a carrier female at $50 \%$ risk of having inherited the abnormal gene may be difficult to diagnose on the basis of clinical signs, causing problems in accurate genetic counselling. XLOA is one of the causes of nystagmus in infancy and is often misdiagnosed as congenital motor nystagmus: examination of the mother, even without an obvious family history, may enable the diagnosis to be made without the need for further investigation.

Most previous studies of the condition have been confined to single large pedigrees or small numbers of families. ${ }^{5,6,14,18,19}$ The aim of this study was to examine a large number of affected families in a systematic fashion to assess and quantify the spectrum of clinical signs and skin histology seen in obligate heterozygotes and at risk females and determine the relative values of clinical examination and skin biopsy in the diagnosis of the carrier state of XLOA.

\section{PATIENTS AND METHODS}

Seventeen multi-generation families affected by XLOA were examined in England and Scotland. Within each kindred a full ophthalmic examination was performed upon all available family members by a single observer (SJC), including assessment of best corrected visual 


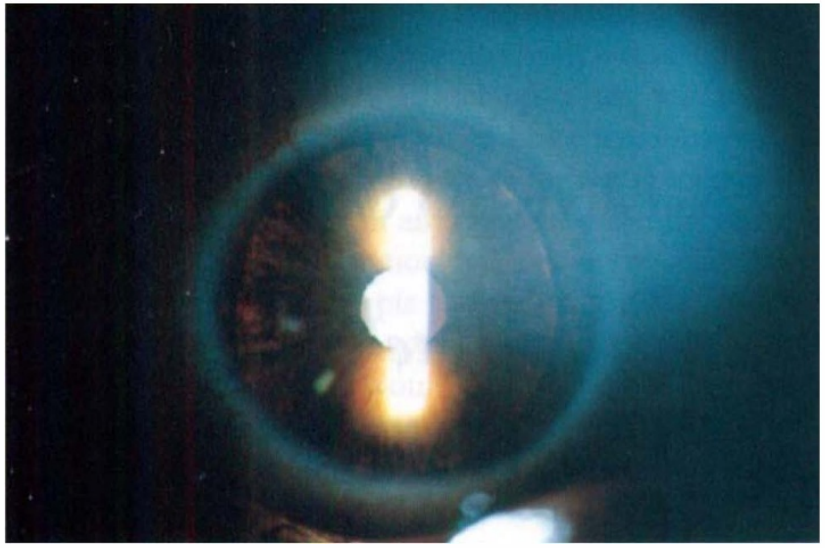

Fig. 1. Iris translucency in an XLOA carrier female showing visible lens periphery (Grade 3 translucency).

acuity, ocular movements, colour vision, slit lamp examination to evaluate iris translucency and ophthalmoscopy. Where possible a skin biopsy was performed in each adult.

Primary diagnostic criteria for affected males were reduced visual acuity, iris translucency, hypopigmented fundi, macromelanosomes on skin biopsy and a family history consistent with X-linked inheritance. In all families at least one male met these criteria. Obligate carriers were diagnosed on the basis of having an affected father, or having an affected son plus an affected brother or other maternal male relative. Signs of the carrier state were classified according to Tables I and II. Iris translucency was assessed by slit lamp microscopy in a darkened room after dark adaptation of the observer, with an axial light beam of maximum brightness directed at the pupil. At risk females were diagnosed as definite carriers on the basis of a. mud-splattered appearance of the fundus with hyperpigmented streaks in the periphery (grade 2 fundus). Some females had pigmentary abnormalities on ophthalmo-

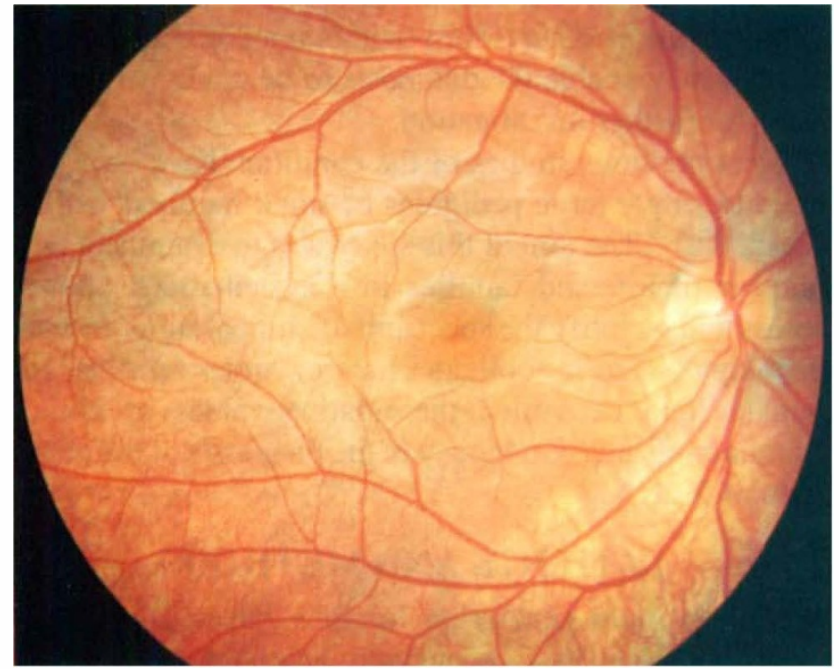

Fig. 2a. scopy, neither typical of XLOA nor absolutely normal, and these were classified as 'uncertain status'.

Skin biopsies, $4 \mathrm{~mm}$ in diameter, were taken from the inner aspect of the forearm under local anaesthesia. Sections were stained with haematoxylin and eosin and by the Masson-Hamperl method. They were examined by a histopathologist (JWG) unaware of the clinical status to determine whether macromelanosomes were present.

\section{RESULTS}

Two hundred and fifteen individuals from seventeen families were examined including 55 affected males, 49 obligate carriers and 64 at risk females. Of the affected males, ten $(18.2 \%)$ had been previously diagnosed incorrectly as having congenital nystagmus. Another, aged 56 years, was unaware of any ophthalmic problem and had visual acuities 6/12 right, 6/9 left, exotropia, grade 3 iris translucency, foveal hypoplasia and macromelanosomes on skin biopsy. His daughter showed typical features of the carrier state and he had two affected nephews. Thirty-two obligate carriers were seen who had affected sons: of these 30 had been unaware of their carrier state before the birth of the affected child, even in the presence of a positive family history.

Obligate carriers. Slit lamp examination was possible in 47 out of 49 obligate carriers. Of these, $37(78.8 \%)$ had grade 3 iris translucency, $4(8.4 \%)$ grade $2,3(6.4 \%)$ grade 1 , and $3(6.4 \%)$ had no defects on transillumination. Fortyfive out of 49 obligate carriers (91.8\%) had grade 2 fundus signs with linear hyperpigmented streaks. In four obligate carriers ophthalmoscopy showed mild peripheral pigmentary stippling (Grade 1 fundus), and in one this was not associated with iris translucency. Skin biopsy demonstrated macromelanosomes in all four cases. Thirty-five

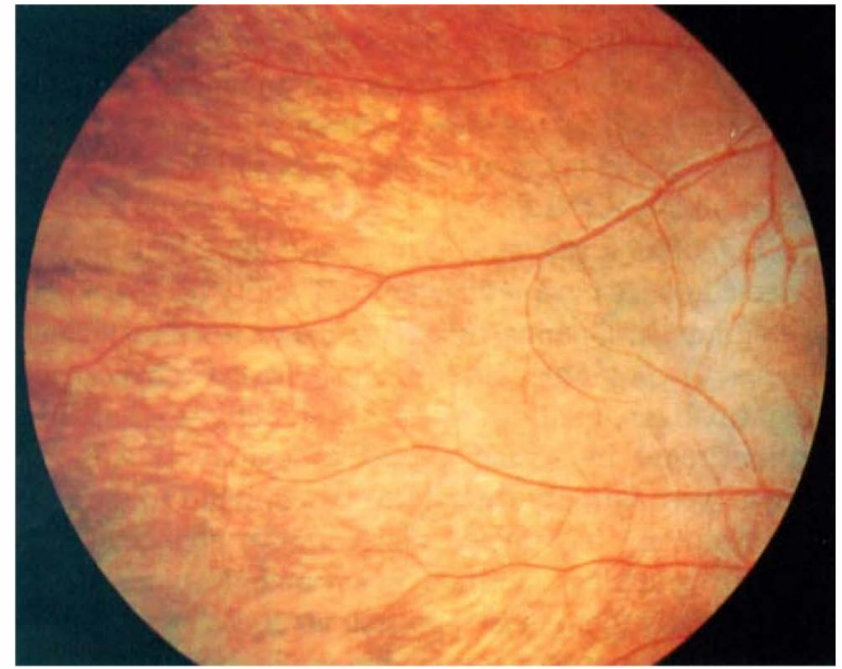

Fig. 2b.

Fig. 2. Colour fundus photograph of an obligate carrier female showing typical 'mud-splattered' fundus appearance with pigmentary stippling at the posterior pole (Fig. 2a) and hyperpigmented streaks in the periphery (Fig. 2b) in the carrier state of XLOA (Grade 2 fundus). 


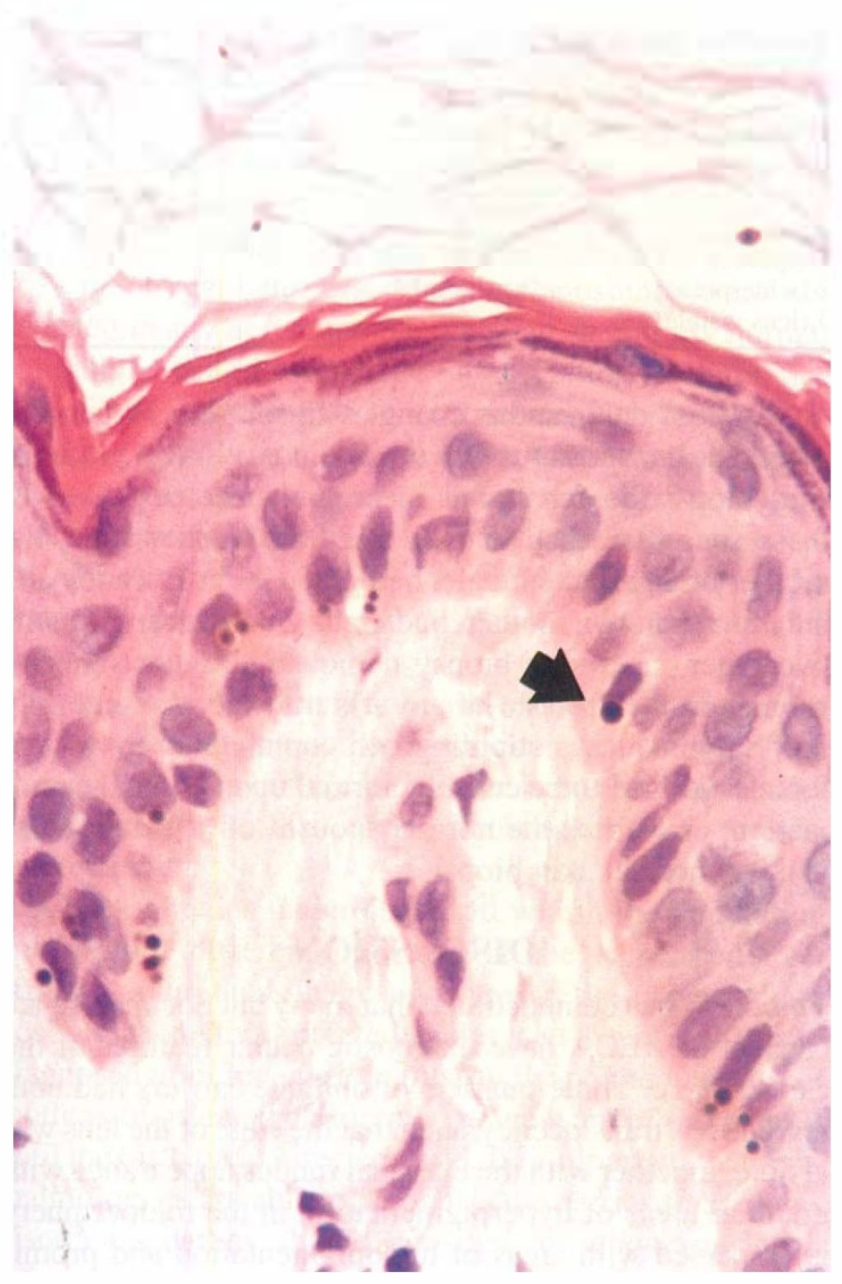

Fig. 3. Macromelanosomes (largest arrowed) as seen by light microscopy in the skin biopsy of an affected male ( $H \& E, X 40)$.

obligate carriers (74.5\%) had both grade 3 iris and grade 2 fundus changes. Forty-seven obligate carriers (95.9\%) had either definite iris (grade 3) or fundus (grade 2) changes. The relationship between iris and fundus signs in those 47 patients where slit lamp and fundus examination was possible is shown in Figure 4.

Even in the presence of hyperpigmented streaks there was a marked variation in the distribution of streaks: in some cases the streaks extended within the macular Table I. Grading of Iris Translucency

\begin{tabular}{ll}
\hline Grade & Description of Iris \\
\hline 0 & No iris translucency \\
1 & A few specks of translucency only \\
2 & Widespread translucency \\
3 & Widespread translucency such that edge of lens visible \\
& around at least $180^{\circ}$
\end{tabular}

Table II. Grading of XLOA Carrier Fundus

\begin{tabular}{|c|c|}
\hline Grade & Description of Fundus \\
\hline 0 & Normal fundus \\
\hline 1 & Possible pigment abnormality \\
\hline 2 & $\begin{array}{l}\text { Mud-splattered appearance with linear hyperpigmented } \\
\text { streaks in periphery }\end{array}$ \\
\hline
\end{tabular}

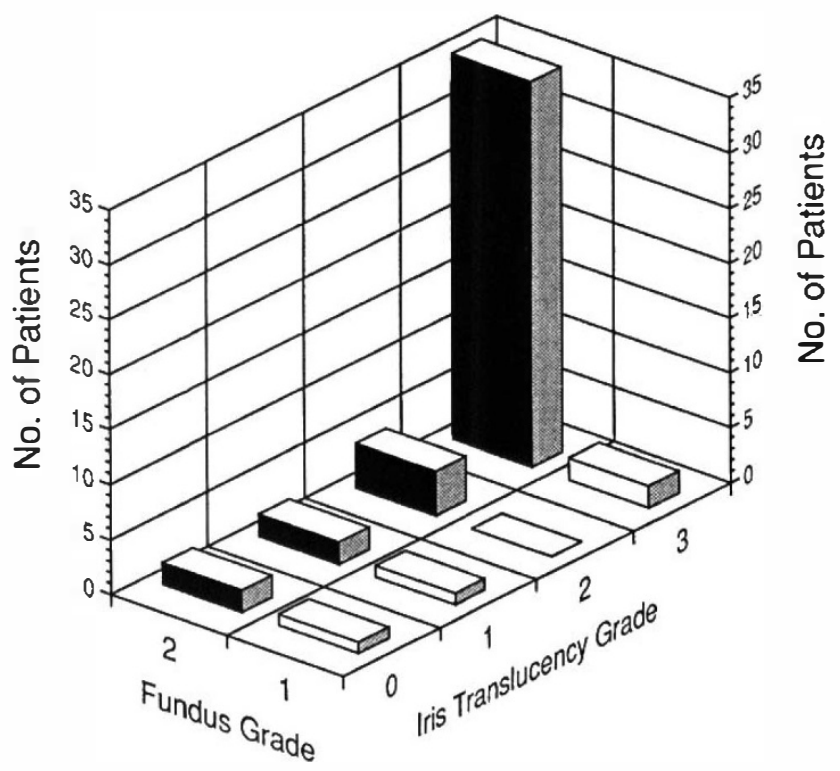

Fig. 4. Correlation of iris and fundus signs in obligate carriers.

arcades whereas in other cases, where the fundus was hypopigmented overall, a few streaks only could be seen in the periphery. In some cases the fundus looked similar to that of an affected male, with marked thinning of the retinal pigment epithelium outside the macular arcades with prominent choroidal vessels, in the absence of myopia, but with a normal foveal reflex (Fig. 5). All obligate carriers had good visual acuity and none had nystagmus.

At risk females. Twenty-seven at risk females $(42.2 \%)$ had definite signs of the carrier state, with mud-splattered fundus appearance and peripheral hyperpigmented streaks, 19 (29.7\%) had normal ophthalmic examinations and 18 (28.1\%) were of uncertain status. In those with definite signs of the carrier state, slit lamp examination and oph-

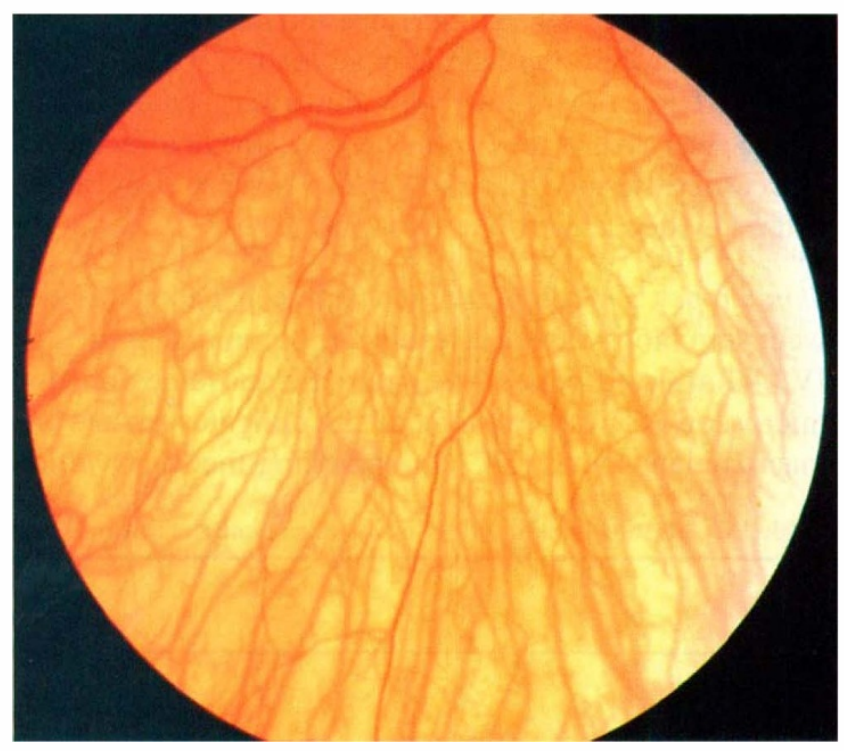

Fig. 5. Colour fundus photograph of an obligate carrier showing marked peripheral hypopigmentation. Marked iris translucency was also present. 


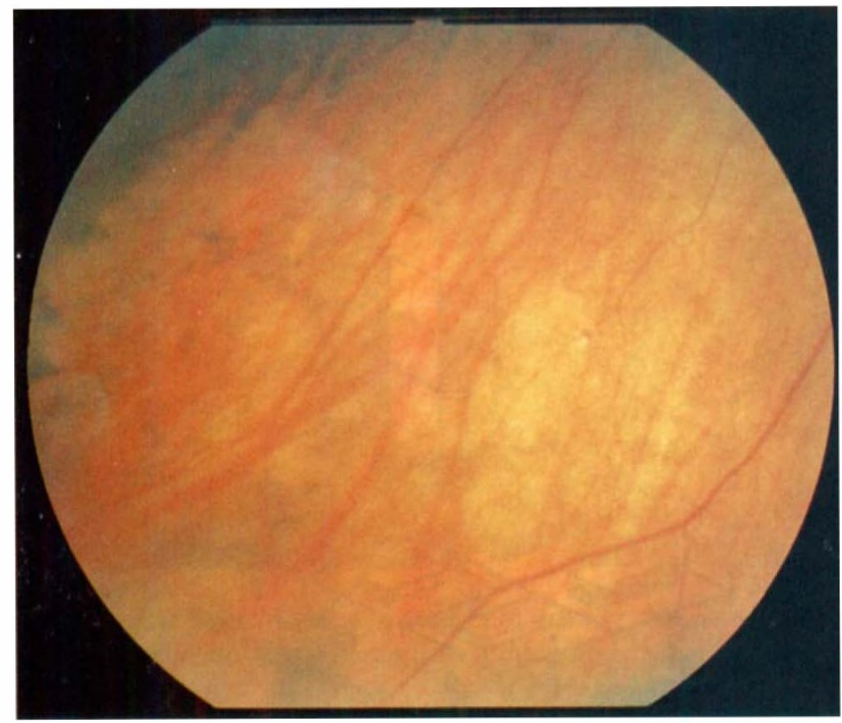

Fig. 6. Colour fundus photograph of an 'uncertain status' female showing peripheral pigmentary abnormality atypical of the carrier state (Grade 1 fundus). No iris translucency was present. Skin biopsy later demonstrated macromelanosomes.

thalmoscopy was possible in 26 cases: 21 had both grade 3 iris translucency $(80.8 \%)$, two had grade 2 iris translucency and three had no iris translucency. In 18 cases mild retinal pigment epithelial abnormalities were noted (Fig. 6), associated in six cases with mild and in four cases with marked iris translucency. On clinical examination alone, these females could neither be diagnosed as being carriers nor definitely normal and were therefore classified as 'uncertain status'.

Unaffected Individuals. Slit lamp microscopy was performed on all family members including unaffected males and normal spouses to determine degrees of iris translucency. Results are shown in Table III. The husband of one obligate carrier had blue irides and marked grade 3 iris translucency, but ophthalmic examination was otherwise normal. Three others (two with blue irides and one mixed blue/brown) had grade 2 translucency: one unaffected son of a carrier was also diabetic. All had normal visual acuity and no evidence of foveal hypoplasia.

Skin Biopsy. One hundred and eighteen patients underwent skin biopsy and the results are summarised in Table IV. All affected males had macromelanosomes on light microscopy. Thirty-two out of 38 obligate carriers had macromelanosomes $(84.2 \%)$, including the four who did
Table III. Iris translucency in unaffected individuals

\begin{tabular}{lccc}
\hline $\begin{array}{l}\text { Iris translucency } \\
\text { (Grade) }\end{array}$ & $\begin{array}{c}\text { Female } \\
\text { spouse } \\
(\mathrm{n})\end{array}$ & $\begin{array}{c}\text { Male } \\
\text { spouse } \\
(\mathrm{n})\end{array}$ & $\begin{array}{c}\text { Unaffected } \\
\text { male } \\
(\mathrm{n})\end{array}$ \\
\hline 0 (none) & 4 & 11 & 24 \\
1 (specks) & 0 & 0 & 2 \\
2 (widespread) & 2 & 0 & 1 \\
3 (lens visible) & 0 & 1 & 0 \\
\hline
\end{tabular}

not have grade 2 fundus changes. In four at risk females where the carrier status was uncertain on clinical grounds, skin biopsy demonstrated macromelanosomes. Two of these four cases had marked iris translucency: one, the mother of an affected male, had grade 3 translucency and the other, her own mother, had grade 2 translucency. In the two other cases skin biopsy demonstrated macromelanosomes in the absence of any iris translucency and only mild pigmentary stippling on ophthalmoscopy. All females judged to be entirely normal upon clinical examination, as well as the normal spouses of affected males (5), had normal skin biopsies.

\section{DISCUSSION}

This study has demonstrated that many but not all obligate carriers of XLOA have diagnostic ocular features of the carrier stage. Three-quarters of obligate carriers had both marked iris translucency, such that the edge of the lens was visible, together with the classical fundus appearance with granular areas of hyperpigmentation in the midperiphery interspersed with areas of hypopigmentation and prominent choroidal vessels. In the periphery the hyperpigmented patches coalesced to form linear streaks. Fine pigmentary stippling was seen at the macula. Iris translucency is found in $10 \%$ of the normal population ${ }^{20}$ and is more common in blue irides and with advancing age. ${ }^{21}$ Although marked iris translucency was seen in over threequarters of obligate carriers in only two cases did it occur without definite fundus changes. Marked iris translucency was occasionally seen in otherwise normal individuals and therefore we would not diagnose the carrier state on iris changes alone. The mud-splattered fundus appearance is a more reliable clinical sign.

Although it can be very easy to diagnose the carrier state in an 'at risk' female, it may be more difficult to exclude a female from being a carrier and thus totally without risk of having an affected child. Twenty-seven of 64 at risk females were identified as carriers on clinical findings, but a further $18(28.1 \%)$ were of uncertain status after clinical

Table IV. Results of skin biopsy in X-linked ocular albinism

\begin{tabular}{llccc}
\hline & & \multicolumn{2}{c}{ Macromelanosomes } & \\
Status & Absent & Present & Total skin biopsies \\
\hline Affected males & & 0 & $35(100 \%)$ & 35 \\
Obligate carriers & & 6 & $32(84.2 \%)$ & 38 \\
'At risk' females & Definite carrier signs & 4 & $13(76.5 \%)$ & 17 \\
& Uncertain status & 7 & 4 & 11 \\
Normal female spouse & No carrier signs & 12 & 0 & 12 \\
\hline
\end{tabular}


examination alone. In four such females skin biopsy demonstrated macromelanosomes: in two the carrier state was suspected due to the presence of marked iris translucency, but in the other two iris translucency was absent. In our experience skin biopsy was useful as a confirmatory test of carrier status, and macromelanosomes were seen in $85 \%$ of obligate carriers. Macromelanosomes were not detected in any at risk female who had an entirely normal ophthalmic examination. The interpretation of skin biopsies, like assessment of the carrier fundus, requires an experienced observer. We would not advocate skin biopsy as a more sensitive means of carrier detection than ophthalmic examination but it may be helpful in the presence of mild clinical signs. The most important factor in the correct diagnosis of the carrier state remains ophthalmoscopy.

Some obligate carriers had mild clinical signs but in all families, except one, other females in the pedigree had classical features of the carrier state, indicating variability in expression of the carrier state rather than genetic heterogeneity.

XLOA remains underdiagnosed within the population and, within different branches of the same pedigree, affected individuals were unaware of the correct diagoosis. One in five affected males had been incorrectly diagnosed until reviewed in this study and the majority of mothers of affected children were unaware of their status until the birth of an affected child. It is important that ophthalmologists are able to recognise the carrier state in this disorder and they should consider the diagnosis in all infant males presenting with nystagmus. In such cases assessment of the mother by slit lamp examination alone is insufficient. Clinical examination will detect most carrier females and although skin biopsy usually demonstrates macromelanosomes, it is not an invariable finding. Some at risk females can pose a diagnostic problem and in these cases, in order to give accurate genetic counselling, molecular genetic diagnostic tests, which are currently under development, will be required.

SJC is in receipt of a Wellcome Vision Research Fellowship. The authors thank the clinicians who referred cases, and Dr S. Bundey, Professor A. Fielder, Professor B. Jay and Dr M. Jay for their help and advice.

\section{REFERENCES}

1. Kinnear PE, Jay B, Witcop CJ Jr: Albinism. Surv Ophthalmol 1985, 30: 75-101.
2. O’Donnell FE Jr, King RA, Green RW, Witcop CJ Jr: Autosomal recessively inherited ocular albinism. Arch Ophthalmol 1978, 96: 1621-5.

3. Nettleship E: On some hereditary diseases of the eye. Trans Ophthalmol Soc UK 1909, 29: 57-198.

4. O'Donnell FE Jr and Green WR: The Eye in Albinism, in Duane TD ed: Clinical Ophthalmology, vol 4. Philadelphia: JB Lippencott, 1989.

5. O'Donnell FE, Hambrick GW, Green WR et al: X-linked ocular albinism. An oculocutaneous macromelanosomal disorder. Arch Ophthalmol 1976, 94: 1883-92.

6. Garner A and Jay BS: Macromelanosomes in X-linked ocular albinism. Histopathology 1980, 4: 243-54.

7. Konrad K, Wolff $\mathrm{K}$, Honigsmann $\mathrm{H}$ : The giant melanosome: a model of deranged melanosome morphogenesis. $J$ Ultrastruct Res 1974, 48: 102-23.

8. Nakagawa H, Hori K, Sato S, Fitzpatrick TB, Martuza RL: The nature of the melanin macroglobule. $J$ Invest Derm 1984, 83: 134-9.

9. Windhorst DB, Zalickson AS, Good RA: Chediak-Higashi syndrome. Hereditary gigantism of cytoplasmic organelles. Science 1966, 151: 81-3.

10. Jimbow K, Szabo G, Fitzpatrick TB: Ultrastructure of giant pigment granules (macromelanosomes) in the cutaneous pigmented macules of neurofibromatosis. $J$ Invest Derm 1973, 61: 300-9.

11. Guerrier CJ, Lutzner MA, Devico V, Prunieras M: An electron microscopical study of the skin of 18 cases of xeroderma pigmentosum. Dermatologica 1973, 146: 211-21.

12. Creel D, O'Donnell FE Jr, Witcop CJ Jr:Visual system anomalies in human ocular albinos. Science 1978, 201: 931-3.

13. Falls HF: Sex-linked ocular albinism displaying typical fundus changes in the female heterozygote. Am J Ophthalmol 1951, 34: 41-50.

14. Gillespie FD and Covelli B: Carriers of Ocular Albinism with and without ocular changes. Arch Ophthalmol 1963, 70: 209-13.

15. Pearce WG, Johnson GJ, Gillan JG: Nystagmus in a female carrier of ocular albinism. J Med Genet 1971, 9: 126-8.

16. Jaeger C and Jay B: X-linked ocular albinism: a family containing a manifesting heterozygote, and an affected male married to a female with autosomal recessive ocular albinism. Hum Genet 1981, 56: 299-304.

17. Cortin P, Tremblay M, Lemagne JM: X-linked ocular albinism: relative values of skin biopsy, iris transillumination and fundoscopy in identifying affected males and carriers. Can J Ophthalmol 1981, 16: 121-3.

18. Johnson GJ, Gillan JG, Pearce WG: Ocular albinism in Newfoundland. Canad J Ophthalmol 1971, 6: 237.

19. Szymanski KA, Boughman JA, Nance WE et al: Genetic studies of X-linked ocular albinism in a large Virginia kindred. Ann Ophthalmol 1984, 16: 183-96.

20. Jay B, Carruthers J, Treplin MCW, Winder AF: Human albinism. Birth defects 1976, 12: 415-26.

21. Norn MS: Iris pigment defects in normals. Acta Ophthalmologica 1971, 49: 887-94. 